\title{
REVIEW OPEN \\ Pathophysiology of environmental enteric dysfunction and its impact on oral vaccine efficacy
}

\author{
Chelsea Marie ${ }^{1}$, Asad $\mathrm{Ali}^{2}$, Kanta Chandwe ${ }^{3}$, William A. PetriJr ${ }^{1}$ and Paul Kelly ${ }^{3,4}$ \\ Environmental enteric dysfunction (EED) refers to a subclinical disorder of intestinal function common in tropical countries and in \\ settings of poverty and economic disadvantage. The enteropathy that underlies this syndrome is characterized by mucosal \\ inflammation and villus blunting mediated by T cell activation. Epithelial cell disruption and microbial translocation drive systemic \\ inflammation. EED in young children is associated geographically with growth failure, malnutrition, and greatly impaired responses \\ to oral vaccines, notably rotavirus and poliovirus vaccines. In this review, we describe the pathophysiology of EED and examine the \\ evidence linking EED and oral vaccine failure. This evidence is far from conclusive. Although our understanding of EED is still \\ sketchy, there is limited evidence of disturbed innate immunity, B cell disturbances including aggregation into lymphoid follicles, \\ and autoantibody generation. Pathways of T cell activation and the possibility of dendritic cell anergy, which could help explain oral \\ vaccine failure, require further work.
}

Mucosal Immunology (2018) 11:1290-1298; https://doi.org/10.1038/s41385-018-0036-1

\section{INTRODUCTION}

It is widely appreciated that intestinal infection is a common cause of diarrheal disease globally, leading to millions of deaths every year. ${ }^{1}$ It is also well known that this enormous burden of disease is attributable to a relatively limited number of pathogens, including protozoa, bacteria, and viruses. ${ }^{2}$ However, recently published data are beginning to reveal a much larger problem of subclinical intestinal infectious disease associated with intestinal inflammation and malnutrition. It is more than two decades since the negative impact of subclinical cryptosporidiosis on child health was described, ${ }^{3}$ and the severe growth deficit due to cryptosporidiosis was defined. ${ }^{4}$ Recently, molecular diagnostic advances have enabled more sensitive detection of enteric pathogens, and it is becoming clear that children in low and middle income countries (LMIC) carry heavy burdens of intestinal infection. ${ }^{5}$ With rotavirus (RV) causing about $37 \%$ of deaths due to diarrhea in children age $<5$ years, ${ }^{6}$ it is hoped that the current global RV vaccination program worldwide will lead to substantial reductions in deaths due to diarrheal disease. Given that most enteric pathogens are transmitted by the fecal-oral route, it would seem logical that water and sanitation (WASH) interventions should reduce enteropathogen burdens. Surprisingly, the evidence to date suggests that efficacy of WASH interventions in reducing diarrhea, malnutrition, or both is lower than would be expected. ${ }^{7}$ Vaccination is the alternative extant strategy for reducing morbidity and mortality due to enteropathogens, and combined oral vaccination against a range of major pathogens would be highly desirable.

\section{THE PROBLEM WITH ORAL VACCINE EFFICACY}

Although oral vaccines are available (Table 1), they work less well in LMIC than they do in industrialized countries. ${ }^{8}$ The live, attenuated cholera vaccine CVD $103-\mathrm{HgR}$ elicited a significant (four-fold or greater) rise in serum vibriocidal antibody in North American adults, but responses to the same vaccine were impaired in Indonesia, Thailand, Peru, and Ecuador. ${ }^{9}$ The same was true of oral RV vaccine: efficacy was $78 \%$ against severe RV diarrhea in Finland, ${ }^{10}$ but only $49 \%$ in Malawi. ${ }^{11}$ RV vaccine efficacy was also reduced in Central America ${ }^{12}$ and Asia. ${ }^{13}$ Madhi et al. ${ }^{11}$ showed that, because of the high incidence of severe disease, a disappointing vaccine efficacy of $61 \%$ still resulted in a substantial vaccine-attributable overall reduction in severe gastroenteritis of 5.0 cases per 100 infant-years. They also compared the severe gastroenteritis episode cases from Malawi and South Africa and found that, although vaccine efficacy was higher in South Africa, there were more episodes (6.7 episodes prevented) of severe RV gastroenteritis per 100 infant-years prevented by vaccination in Malawi than in South Africa (4.2 episodes prevented). Even though the efficacy of RV vaccine is low, it is therefore still of value in the populations most heavily affected. Oral polio vaccine (OPV) is also much less efficacious in developing countries, ${ }^{14,15}$ and in recent campaigns in northern India up to 20 doses have been administered per child. So, although oral vaccines are available for some enteric infections, and can be successful in reducing disease burden across the globe, improved efficacy would be very valuable.

The reasons for the impaired efficacy of oral vaccines in LMIC are not yet clear. Several possible factors may help explain this phenomenon. Possibilities include interference from the high titers of antibody in maternal breast milk, ${ }^{16}$ nutritional factors such as vitamin A deficiency, ${ }^{17}$ and environmental enteropathy (EE). ${ }^{18}$ $\mathrm{EE}$ is an asymptomatic inflammatory disorder of the proximal small intestine, ${ }^{8}$ which underlies environmental enteric dysfunction (EED). At least for polio virus type 1, it is highly likely that

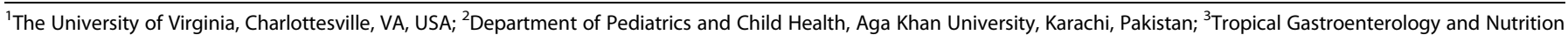
group, University of Zambia School of Medicine, Lusaka, Zambia and ${ }^{4}$ Barts \& The London School of Medicine, Queen Mary University of London, London E1 4AT, UK Correspondence: Paul Kelly (m.p.kelly@qmul.ac.uk)
}

Received: 8 December 2017 Revised: 3 April 2018 Accepted: 11 April 2018

Published online: 9 July 2018 
Table 1. Commercially available oral vaccines

\begin{tabular}{|c|c|c|c|c|}
\hline Vaccine type & Disease & Vaccine constituents & Protection & Commercial name \\
\hline Live attenuated & Typhoid & S. typhi (Ty21a) & $67 \%$ over 3 years & Vivotif \\
\hline Live attenuated & Cholera & V. cholera (CVD103-HgR) & $80-90 \%$ & Vaxchora \\
\hline Live attenuated & Rotavirus & Attenuated virus & $85-100 \%$ & Rotarix \\
\hline Live attenuated & Rotavirus & Human-bovine reassortant viruses & $74 \%$ & RotaTeq \\
\hline Inactivated & Cholera & Heat-killed V. cholerae+CTB & $80-90 \%$ & Dukoral \\
\hline Inactivated & Cholera & V. cholerae (Biv-WC) & $\begin{array}{l}90 \% \text { at } 10 \text { days; protection } \\
\text { at later times unknown }\end{array}$ & Shanchol, Euvichol \\
\hline
\end{tabular}

interference by concurrent infections such as non-polio enteroviruses contribute substantially to impaired vaccine efficacy, and efficacy is also lower in the presence of diarrhea. ${ }^{15}$ There is also evidence that strain variation may contribute to reduced efficacy, at least for RV. ${ }^{19}$ Counter-intuitively, Helicobacter pylori infection, which is common in those populations where oral vaccines are less efficacious, seems unlikely to explain reduced vaccine immunogenicity as there is some evidence that it actually increases it. ${ }^{20}$

\section{OTHER CONSEQUENCES OF EED}

Malnutrition, manifested as stunting and/or acute wasting, is a major factor behind child mortality and inability of children to reach their full neurocognitive potential in resource-poor settings. ${ }^{21}$ Stunting affects 165 million children globally. ${ }^{22}$ Stunting is defined as failure of linear growth, which is often due to chronic, insidious nutritional deficiencies. Wasting is a loss of weight due to a more acute illness, often triggered by an infectious disease. Studies reveal that malnutrition is not just a lack of food issue but is also in large part due to EED. ${ }^{23}$ Absorption of critical micronutrients may also be compromised in children with EED. A study in Malawi showed that net zinc absorption after a challenge dose was negatively correlated with lactulose:mannitol ratio, which is a marker of increased gut permeability induced by EED. ${ }^{24}$ This may be true with other critical micronutrients also. ${ }^{22}$

\section{THE UNDERLYING CAUSES OF EED}

This is an area of real uncertainty. It has been known for several decades that EED is seasonal ${ }^{25}$ and reversible. ${ }^{26-28}$ Emerging evidence suggests that children in LMIC have very high burdens of intestinal infectious disease, ${ }^{5}$ not enough to cause diarrhea but probably enough to induce epithelial damage. It is absolutely plausible, but not proved, that this is the principal driver of enteropathy. The strongest evidence to date that enteropathogen burden contributes to pathogenesis is that azithromycin ameliorates some biomarkers of enteropathy, though it does not significantly improve OPV responses. ${ }^{29}$ The role of malnutrition is uncertain, but micronutrient trials have shown only modest benefits. ${ }^{30,31}$ In Malawian children with stunting, amino acids were found to be generally $\operatorname{low}^{32}$ and $\omega-3$ and $\omega-6$ polyunsaturated fatty acids were also reduced in recent metabolomic studies. ${ }^{33}$ Increasing data suggest that the microbiota constitutes a critical influence on the development of the gut and its mucosal immune system. Evidence from Malawi implicates dysbiosis (a flora altered in composition and usually reduced in complexity) in EED, ${ }^{34,35}$ though whether dysbiosis initiates EE or merely perpetuates it is an unresolved question. Composition and function of the gut microbiota of 2-3-year-old children could be linked with the child and mother's genetically determined secretor status, presumably mediated by associated alterations in host glycans and breast milk-associated human milk oligosaccharides ${ }^{36}$

\section{PATHOLOGY AND PATHOPHYSIOLOGY OF EED}

In populations affected by EED, measurements of villus height or intestinal permeability vary continuously over a wide range, but the distributions of villus height ${ }^{25,37,38}$ and permeability ${ }^{39}$ measurements are reduced compared to populations in industrialized countries. There are disturbances in multiple domains of pathophysiology such as morphological change, malabsorption, mucosal inflammation, microbial translocation (MT), systemic inflammation, and changes in the microbiome. ${ }^{40-42}$ Do these reflect multiple pathophysiological processes? Are there specific derangements that are most clearly associated with failure of oral vaccines or with growth failure? If specific features of pathophysiology can be associated with specific outcomes, then therapy could be better targeted to the desired effect.

The hallmark of the enteropathy of EED is villus blunting, which means that in histological sections villus height is reduced and villus width increased (Fig. 1). There is a spectrum of morphological change, ranging from subtle increases in lamina propria infiltrates to total villus atrophy. ${ }^{25,38}$ It is not possible to specify an absolute value of villus height that is normal, partly in view of the paucity of morphometric data available from industrialized countries free of EED. ${ }^{38}$

Increased intestinal permeability, measured by monosaccharide and disaccharide probes, is also considered a diagnostic hallmark of EED. ${ }^{39,43,44}$ The principle of the test is that larger sugars (such as lactulose) cross the epithelium paracellularly, through dysfunctional tight junctions, while smaller monosaccharides (such as mannitol and rhamnose) are absorbed transcellularly and reflect the absorptive capacity of the epithelium. ${ }^{45}$ This test does not discriminate between opening up of the tight junctions, ${ }^{46,47}$ which would allow only small molecules to cross the epithelium, and larger defects such as those recently identified using confocal laser endomicroscopy (CLE). CLE permits imaging of the leakage of fluorescein from systemic circulation into the gut lumen. In adults with EED, CLE identified extensive leakage into the lumen focused at villus tips, suggesting that microerosions caused by disordered epithelial cell shedding may be an important factor underlying increased intestinal permeability in EED. ${ }^{37}$

Barrier failure is associated with increased MT from the lumen to the systemic circulation. Although the correlation between the two is not close, ${ }^{48}$ biomarkers of translocation and inflammation did correspond across different patient groups in Zambia (Fig. 2). MT can exacerbate chronic inflammatory states, ${ }^{49}$ including EED. ${ }^{50}$ Biomarkers of MT include: lipopolysaccharide (LPS), a component of bacterial cell walls; the soluble LPS co-receptor CD14 (sCD14), which is upregulated by LPS; and antibodies to the core LPS core antigen (EndoCAb), which decrease after LPS binding (Table 2). Multiple other biomarkers are available that measure derangements in multiple domains of pathology, including permeability, enterocyte injury, and mucosal and systemic inflammation (Table 2). In adults with EED, intestinal permeability (as measured by CLE) was correlated with plasma LPS concentrations. ${ }^{37}$ Moreover, MT increased pro-inflammatory cytokines such as tumor 

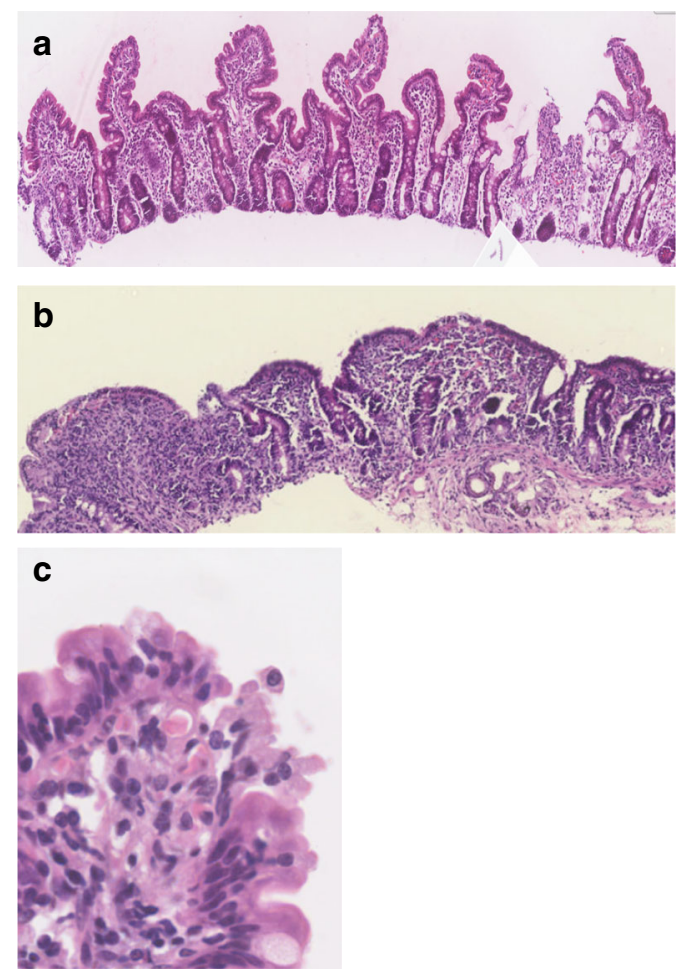
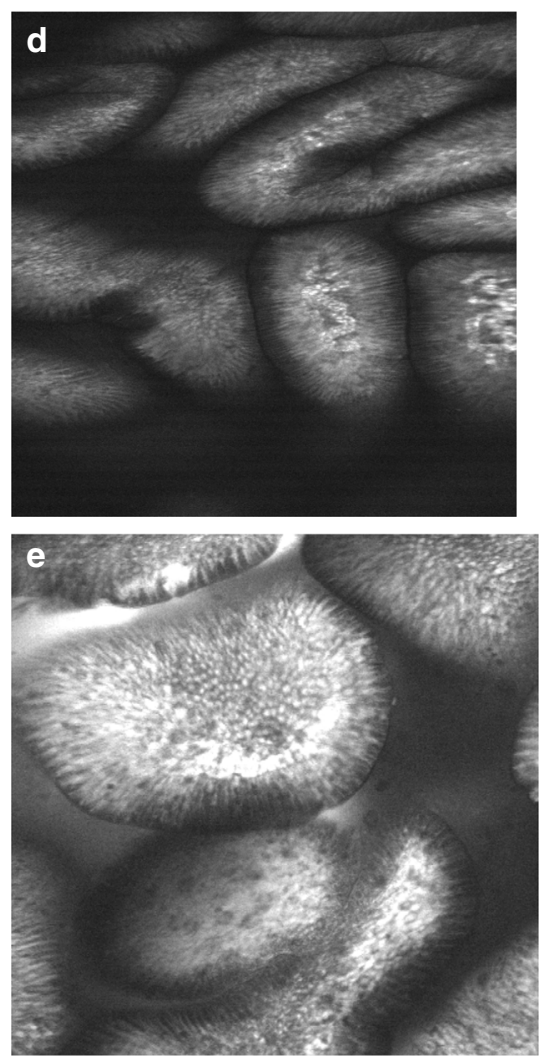

Fig. 1 Hematoxylin and eosin-stained sections of small intestinal biopsies from Zambian adults with EE and children with more severe malnutrition showing a moderate enteropathy (villus height:crypt depth $<1.0$ ) with pronounced inflammatory cell infiltrate, $\mathbf{b}$ total villus atrophy with lymphoid aggregates in the lamina propria, c higher magnification of epithelial lesion with several cells in detachment and exposure of basement membrane to the luminal stream. Imaging using confocal laser endomicroscopy ${ }^{37}$ shows $\mathbf{d}$ normal mucosa with all circulating (white) fluorescein contained by the epithelial barrier, and e leakage of fluorescein into the lumen leading to opacification at the top of the image

necrosis factor-a (TNFa), interferon- $\gamma$ (INFY), interleukin (IL)-1 $\beta$, and IL-13.

TNFa can directly modulate barrier function via regulation of epithelial cell shedding and actin cytoskeleton via activation of myosin light chain kinase (MLCK). Mice with constitutively active MLCK develop chronic subclinical mucosal immune activation, as measured by increased numbers of lamina propria $\mathrm{CD}^{+}{ }^{+} \mathrm{T}$ cells and increased production of IFN- $\gamma$ and TNF-a. Despite this, mice grew normally and did not develop spontaneous colitis, though the severity and onset of immune-induced colitis was significantly worse. . $^{1}$

\section{MUCOSAL IMMUNOLOGY IN EED}

The development of mucosal immunity after oral vaccination depends on many immune cell interactions. Briefly, mucosal dendritic cells (DCs) present antigen to T cells and both T cells and DCs can stimulate $B$ cells to mature, traffic to the intestine, and produce secretory immunoglobulin $A(s \lg A)$. In EED, repeated exposure to enteric pathogens is hypothesized to induce a state of chronic immune activation at the intestinal epithelium. ${ }^{52}$ The mucosal immune response in EED is understudied owing to safety and ethical concerns of invasive intestinal biopsies. A single study comparing immune infiltrates in Gambian children with EED to UK controls identified profound alterations in the mucosal immune system. ${ }^{53}$ Similar immune alterations in the intestinal mucosa have also been described in adults with EED. ${ }^{54}$ Further, a recent transcriptomic study of cells isolated from fecal samples of children found that the 12 transcripts correlated with EED severity were related to mucosal immune responses including: chemokines that stimulate $\mathrm{T}$ cell proliferation, $\mathrm{Fc}$ fragments of multiple immunoglobulin families, IFN-induced proteins, activators of neutrophils and B cells, and mediators that dampen cellular responses to hormones. ${ }^{55}$ More detailed studies of the alterations in immune cell populations and their impact of these oral vaccine response in EED are important areas for future investigation.

\section{T CELL DERANGEMENTS IN EED}

Mucosal $\mathrm{T}$ cells are critical mediators of intestinal immunity. Increased densities of intraepithelial (intraepithelial lymphocytes (IELs)) and lamina propria (LP) T cell populations have been observed in intestinal biopsies of children with EED, which was subsequently referred to as a T cell-mediated enteropathy. ${ }^{53,54}$ Expression of CD69 and HLA-DR were increased in T cells in the mucosa in adults in Zambia compared to South African controls, and numbers of IELs were increased. ${ }^{54}$

\section{Intestinal IELs}

IELs constitute a large and diverse population of lymphoid cells that reside between intestinal epithelial cells (IECs) and mediate barrier protection and homeostasis. A study of children with $\mathrm{EE}$ found elevated numbers of IELs with increased frequencies of both $\mathrm{CD}^{+}$perforin and TCR $\delta^{+}$IELs compared to UK controls. ${ }^{53}$ IELs arise from distinct lineages. In EE, the increased IEL counts could arise from increased antigenic exposure leading to accelerated accumulation of "induced" IELs (originating from conventional TCRa $\beta$ activated in the periphery). However, the observation of increased $\mathrm{TCR} \gamma \delta^{+}$cells is also of note, as this "natural" IEL subset traffics to the gut directly upon maturation 

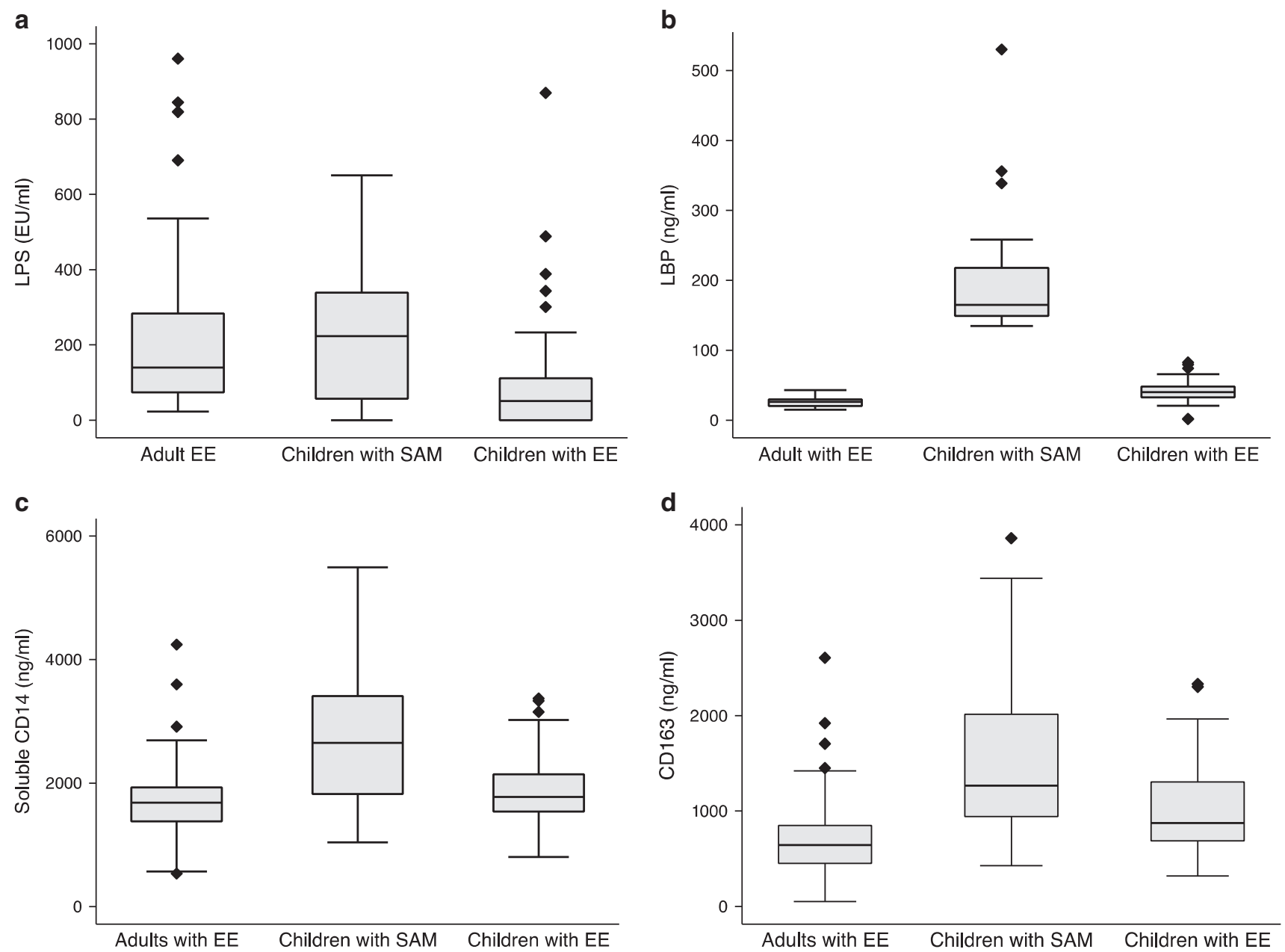

Fig. 2 Biomarkers of microbial translocation and systemic inflammation in plasma from three groups: adults with EED, children with severe acute malnutrition (SAM), and children with EED (data from Amadi et al. ${ }^{38}$ ). Biomarkers shown are a plasma lipopolysaccharide (LPS); b plasma LPS binding protein (LBP); c plasma soluble CD14; and d plasma CD163, a marker of Kupffer cell response to microbial translocation ${ }^{50}$

and may recognize auto-antigens. ${ }^{56}$ Both subsets of IELs are not only important for pathogen clearance, homeostasis, and tissue repair but can also promote pathology via excessive cytotoxicity and inflammatory responses. In celiac disease (CD), IEL-mediated direct cytotoxic activity toward IECs increases intestinal pathology. ${ }^{57-59}$ A critical feature of IELs is rapid T cell receptor (TCR)dependent and/or TCR-independent activation that can shape downstream immune response. ${ }^{60,61}$ As such, IEL activation has the potential directly or indirectly to shape the development of mucosal immunity to oral vaccination, though the implications for antigen specificity of responses are not understood. One example of IEL participation in vaccine responses is suggested by the recent finding that secreted factors from activated IELs upregulated antiviral IFN-responsive genes in IECs and increased resistance to norovirus infection in vitro. ${ }^{62}$ This study raises the possibility that, in the context of EED, high numbers of activated IELs could indirectly hinder vaccine efficacy by inducing IEC resistance to infection with live-virus vaccines.

Regulatory and effector T cells

In EED, repeated enteric infections are hypothesized to increase $T$ cell recruitment and activation in the intestine. Increased $T$ cell activation has also been documented in Zambian adults via expression of the markers CD69 or HLA-DR. ${ }^{54}$ Likewise, children with EED have higher frequencies of both regulatory (transforming growth factor- $\beta$ and IL-10) and pro-inflammatory (IFN- $\gamma$ and TNF- $\alpha$ ) cytokine-secreting cells in the LP. ${ }^{53}$ In healthy individuals, tissue $T$ cells were predominantly naive and regulatory in infants, with specialized tissue-resident effector memory populations accumulating with age. ${ }^{63,64}$ Tissue regulatory $T\left(T_{\text {Reg }}\right)$ cells suppress endogenous T cell activation and can suppress harmful inflammation and promote epithelial repair. Children with enteropathy had 4-5-fold more $\mathrm{LP} \mathrm{CD3}^{+} \mathrm{T}$ cells and 15-30-fold more $\mathrm{CD} 25^{+} \mathrm{T}$ cells relative to UK controls. Of note, $\mathrm{CD}_{2} 5^{+}$cells were decreased in children with the most severe malnutrition. ${ }^{53}$ As CD25 is expressed by $T_{\text {regs, }}$ as well as by activated $T$ cells, a more detailed analysis of $T$ cell phenotype and function is needed to understand the origin and function of $\mathrm{CD} 25^{+}$cells in EED and vaccine response. Overall, limited histologic data suggest that increased $T$ cell recruitment and activation in intestinal tissue is a hallmark of EED, and understanding the origin and function of T cell subsets in EED will likely be helpful for understanding reduced oral vaccination efficacy.

\section{B CELL DERANGEMENTS}

A key feature of oral vaccination is the induction of mucosal memory responses mediated by $B$ cells residing in gut-associated lymphoid tissue. Children with EED had 2-3 times more B cells in the LP compared to UK controls, whereas mature plasma cells $\left(\right.$ syndecan $-1^{+}$) were $25-30$ times higher. ${ }^{53}$ In EED, unlike in CD which otherwise has similar histology, B cell aggregates are found 
Table 2. Commonly used biomarkers of EED

\begin{tabular}{|c|c|c|c|}
\hline Biomarker & Source & Interpretation & Ref. \\
\hline \multicolumn{4}{|l|}{ Intestinal absorption } \\
\hline \multicolumn{4}{|c|}{ None in widespread use, stable isotope approaches being evaluated } \\
\hline \multicolumn{4}{|c|}{ Intestinal "leak"'and permeability; tight junction disruption } \\
\hline Dual (or quadruple) sugar tests & $\begin{array}{l}\text { Differential absorption of sugars } \\
\text { based on size }\end{array}$ & $\begin{array}{l}\text { Increased lactulose permeation in relation to monosaccharide } \\
\text { absorption (e.g., rhamnose, mannitol) reflects increased } \\
\text { permeability }\end{array}$ & $\begin{array}{l}39,43 \\
44\end{array}$ \\
\hline $\begin{array}{l}\alpha-1 \text {-Antitrypsin (AAT) in stool or } \\
\text { duodenal aspirates }\end{array}$ & Protease inhibitor & $\begin{array}{l}\text { Not synthesized in the gut, AAT in stool reflects protein loss and } \\
\text { increased permeability. Can be increased by infection }\end{array}$ & 91 \\
\hline $\begin{array}{l}\text { Claudin-2, claudin-4, claudin }-15 \text {, } \\
\text { zonulin }\end{array}$ & $\begin{array}{l}\text { Tight junctions between } \\
\text { epithelial cells }\end{array}$ & $\begin{array}{l}\text { Increased release into blood or urine may reflect breach of } \\
\text { barrier function; dysregulated in epithelial cells }\end{array}$ & $\begin{array}{l}37,38 \\
92\end{array}$ \\
\hline \multicolumn{4}{|l|}{ Enterocyte mass, turnover, or injury } \\
\hline Citrulline & Enterocytes & Correlated with enterocyte mass, surface area & 93 \\
\hline $\begin{array}{l}\text { Regenerating proteins (Reg1a and } \\
\text { Reg1b) }\end{array}$ & Paneth cells, intestinal crypt cells & $\begin{array}{l}\text { Released in response to injury. Antiapoptotic, stimulates tissue } \\
\text { regeneration, and cell proliferation }\end{array}$ & 94 \\
\hline $\begin{array}{l}\text { Intestinal fatty acid binding protein (I- } \\
\text { FABP) }\end{array}$ & $\begin{array}{l}\text { Epithelial protein located at villus } \\
\text { tips }\end{array}$ & $\begin{array}{l}\text { Short half-life in circulation, high levels indicate recent intestinal } \\
\text { injury }\end{array}$ & 37,38 \\
\hline Glucagon-like peptide 2 (GLP-2) & Enteroendocrine cells & $\begin{array}{l}\text { Hormonal response to malabsorption aimed at epithelial repair; } \\
\text { interpretation not yet clear }\end{array}$ & 37 \\
\hline \multicolumn{4}{|l|}{ Microbial translocation } \\
\hline Lipopolysaccharide (LPS) & $\begin{array}{l}\text { Component of Gram-negative } \\
\text { bacteria }\end{array}$ & Increased during microbial translocation & 50 \\
\hline $16 \mathrm{~S}$ rRNA gene DNA in blood & Component of all prokaryotes & Increased during microbial translocation & 50 \\
\hline LPS-binding protein & $\begin{array}{l}\text { Released by mononuclear cells on } \\
\text { LPS binding }\end{array}$ & Increased during microbial translocation & 50 \\
\hline Endotoxin core antibodies & Antibody response to LPS & Increased during microbial translocation & 53 \\
\hline \multicolumn{4}{|l|}{ Mucosal inflammation } \\
\hline Neopterin (NEO) in stool & Macrophages and dendritic cells & Produced in response to IFN- $\gamma$; marker of inflammation & 91 \\
\hline Myeloperoxidase (MPO) in stool & Neutrophils & Bacterial killing; marker of inflammation & 91 \\
\hline Calprotectin in stool & Neutrophils & Indicator of gut damage but can be high in healthy infants & 95 \\
\hline Kynurenine-tryptophan ratio (KTR) & $\begin{array}{l}\text { Indolamine 2,3-dioxygenase } \\
\text { activity }\end{array}$ & $\begin{array}{l}\text { Immune activation, though tissue responsible for altered ratio } \\
\text { not yet clear }\end{array}$ & 96 \\
\hline \multicolumn{4}{|l|}{ Systemic inflammation } \\
\hline Soluble CD14 (sCD14) in blood & $\begin{array}{l}\text { Released by mononuclear cells on } \\
\text { LPS binding }\end{array}$ & Soluble LPS receptor & 37,38 \\
\hline $\begin{array}{l}\text { Pro-inflammatory cytokines: (IFN- } \gamma \text {, } \\
\text { TNF- } \alpha, \text { IL- } 6, \text { IL-10) }\end{array}$ & $\begin{array}{l}\text { Signaling cytokines that activate } \\
\text { immune cells }\end{array}$ & Inflammation & 50 \\
\hline $\begin{array}{l}\alpha \text {-1-acid glycoprotein, C-reactive } \\
\text { protein (CRP), ferritin }\end{array}$ & Acute-phase proteins & Inflammatory response & 37 \\
\hline
\end{tabular}

in the LP (J Turner, personal communication). Murine studies have shown that small intestinal B cell aggregates are induced by environmental signals, including the microbiota ${ }^{65}$; however, the origin of $B$ cell aggregates in EED and their impact on memory cell formation and IgA production is still unknown.

\section{Secretory $\lg A$}

slgA is the major antibody at the intestinal mucosa and is a critical component of immunity induced by oral vaccines. In oral vaccination, mucosal DCs present antigen to $T$ cells and both $T$ cells and DCs stimulate B cells to mature and produce slgA. B cells then enter circulation and traffic to the LP differentiating into long-lived plasma cells that release slgA dimers. IgA dimers can act in the LP, in the epithelium against intracellular pathogens during translocation, or in the intestinal lumen.

The induction of primary $\lg A$ responses and the duration of memory responses are critical for immunity induced by oral vaccination. IgA memory B cells can be detected in peripheral blood following oral cholera vaccination but have a relatively short duration in the circulation relative to IgG memory $\mathrm{B}$ cells. ${ }^{66,67} \mathrm{~A}$ recent study in Bangladesh found that robust circulating $\lg \mathrm{A}$ responses were induced by a single dose of oral cholera vaccine in adults and toddlers but not in infants. ${ }^{68}$ In contrast, the responses to a second dose of cholera vaccine in infants were similar to older children and adults. Plasma IgA peaked on day 5 after vaccination and fecal slgA responses were detected at day 7 , suggesting that plasma IgA decreases as memory B cells migrate to the mucosa. ${ }^{68}$ One possible explanation for the lack of a response in infants is that natural exposure in older individuals primes an enhanced response to the first dose of oral vaccine. We are not aware of studies of total $\lg A$ or slgA in EED nor have studies yet explored a possible effect of vitamin A deficiency on vaccine efficacy through effects on the polymeric Ig receptor as has been demonstrated in vitro. ${ }^{69}$

The duration of intestinal $\lg \mathrm{A}$ memory $\mathrm{B}$ cells induced by oral $E$. coli vaccination is least $1-2$ years in healthy adults. ${ }^{70}$ Recent evidence suggests chronic pathogen exposure could impact the development and duration of slgA responses to oral vaccination in EED. In a mouse model of transient bacterial colonization, a highly specific memory lgA response was maintained even after bacterial 


\begin{tabular}{|lllll|}
\hline Table 3. & Antimicrobial peptides in the gut & & \\
\hline Peptide & Gene & Cellular source & Alteration in EED & Ref. \\
\hline Human defensin 5 & DEFA5 & Paneth cells & Reduced & 77 \\
Human defensin 6 & DEFA6 & Paneth cells & Reduced & 77 \\
Lysozyme & LYZ & Paneth cells & Unknown but increased in celiac and inflammatory bowel diseases & 97 \\
$\alpha 1$-antitrypsin & SERPINA1 & Paneth cells & Unknown & - \\
hBD1 & DEFB1 & Enterocytes & Unknown & - \\
hBD2 & DEFB4 & Enterocytes & Reduced & 78 \\
hBD3 & DEF103B & Enterocytes & Unknown & - \\
LL37 & CAMP & Enterocytes & Reduced & 78 \\
\hline
\end{tabular}

clearance but was rapidly replaced upon exposure to another species of bacteria. ${ }^{71}$ In the context of EED, it is plausible that increased exposure to enteric pathogens results in faster attrition of vaccine-induced $\lg A$ secreting cells as slgA responses adapt to luminal antigen exposure.

A recent study found that children with severe acute malnutrition had increased concentrations of two celiac auto-antibodies: tissue transglutaminase and deamidated gliadin peptides. ${ }^{38}$ Though within normal ranges, auto-antibody concentrations were inversely correlated with villus height and positively correlated with the systemic inflammatory marker LPS-binding protein. Thus it appears that autoreactivity may exacerbate (or possibly just reflect) mucosal pathology in EED as well as in $C D .{ }^{38} \mathrm{CD}$ is a T cellmediated enteropathy with some similar histological features to EE. An early study investigating the efficacy of OPV found subnormal IgA responses to OPV in CD patients ${ }^{72}$; however, a subsequent study found the opposite, with celiac patients producing significantly more IgA than controls. ${ }^{73}$ Thus it remains unclear whether secretory $\lg A$ production is altered more broadly in $\mathrm{T}$ cell-mediated enteropathies.

\section{DERANGEMENTS}

To our knowledge, there are no data on DC populations in EED or on their function. Children with severe acute malnutrition were found to have fewer DCs than healthy children in one study from Zambia, and fully $17 \%$ of children had anergic $\mathrm{DCs},{ }^{74}$ meaning that DCs from malnourished children showed reduced HLA-DR expression, failure of secretion of IL-12, and failure to drive T cell proliferation. This phenomenon was associated with endotoxemia. ${ }^{74}$ As endotoxemia due to MT is a dominant feature of the pathophysiology of EED (Fig. 2), it would be reasonable to propose that DCs may be dysfunctional in EED, but this has not been demonstrated directly.

\section{INNATE IMMUNE DERANGEMENTS}

Innate immunity is a broad term that encompasses cellular and soluble host defense mechanisms, which do not require anamnestic (learned) responses. Innate immunity may be modulated by recent exposure, often referred to as "trained immunity." There is little information about trained immunity in the gut, especially in the context of EED, and this will not be considered further here.

Cellular responses include the activity of polymorphonuclear leucocytes (neutrophils, eosinophils, and basophils) and macrophages. To our knowledge there are no data on mucosal populations of these cells in EED nor on functional capacity. However, there is an indirect evidence for disturbances of these cells in EED. In an analysis of the fecal transcriptome in children with EED, several gene ontology pathways were identified that suggest disturbed neutrophil function. ${ }^{55}$
Soluble mediators of innate defense include antimicrobial peptides $^{75}$ and C-type lectins such as mannose-binding lectin. ${ }^{76}$ Antimicrobial peptides expressed in the gut are summarized in Table 3. There is evidence from several studies in Zambian adults with EED that expression of Paneth cell defensins is reduced compared to adults in the UK, ${ }^{77,78}$ but these studies have not been conducted in other settings and the generalizability of these findings is uncertain. Could this alteration in antimicrobial peptide expression help explain vaccine under-performance? Intriguingly, $\beta$-defensins appear to have adjuvant properties, ${ }^{79-81}$ but again firm data are needed concerning their potential role in contributing to vaccine responses.

\section{COULD ENTEROPATHY PLAUSIBLY EXPLAIN POOR ORAL VACCINE RESPONSES?}

Responses to oral RV vaccine were associated with fecal markers of inflammation in Nicaragua. ${ }^{82}$ Vaccine responses to oral RV vaccine were attenuated in children with malnutrition and diarrhea in Bangladesh, ${ }^{83}$ but this was not found in an earlier study from Brazil and Venezuela. ${ }^{84}$ More recently, the PROVIDE study demonstrated that OPV and RV vaccine responses were negatively impacted by EED, as measured by fecal biomarkers. ${ }^{85}$ Children with evidence of EED at the time of vaccination had lower Rotarix $^{\circledR}$ vaccine response (plasma $\lg \mathrm{A}$ ) and Rotarix ${ }^{\circledR}$ protection from RV diarrhea than those without (Fig. 3). In contrast, EED did not affect the response to tetanus, pertussis, diphtheria, Haemophilus influenza type B, or measles vaccines (Fig. 3). Biomarkers of EED were also negatively associated with linear and ponderal growth to age 1 year. Children with EED also had lower serum-neutralizing antibody responses to OPV (Fig. 3). ${ }^{85}$ Further work suggests that non-polio enterovirus and Campylobacter infection at the time of vaccination may have mediated the poor responsiveness. ${ }^{86}$

A study of oral cholera vaccine efficacy in older children with EED in Bangladesh found contradictory associations between plasma and fecal biomarkers of EED and vaccine responses. In this study, fecal myeloperoxidase (MPO) and plasma sCD14 were positively associated with the development of plasma antibody responses to vaccination, whereas fecal AAT and plasma EndoCab were negatively associated with cholera toxin-specific $T$ cell responses, including IL-10 production. ${ }^{87}$

In a large $(n=754)$ trial in India of azithromycin given from 11 to 14 days before vaccination, the intervention had no effect on OPV responses. ${ }^{29}$ It did, however, reduce fecal MPO by $26 \%$ and $a_{1}$-antitrypsin by $19 \%$, suggesting that enteropathy and vaccine responses can be dissociated.

In a randomized controlled trial of a synbiotic in India, the combination of Lactobacillus plantarum plus fructooligosaccharide was found to reduce sepsis and death in Indian infants. ${ }^{88}$ As the intervention has its effects in the gut, it is likely that the synbiotic has its effects on reducing MT, perhaps by 


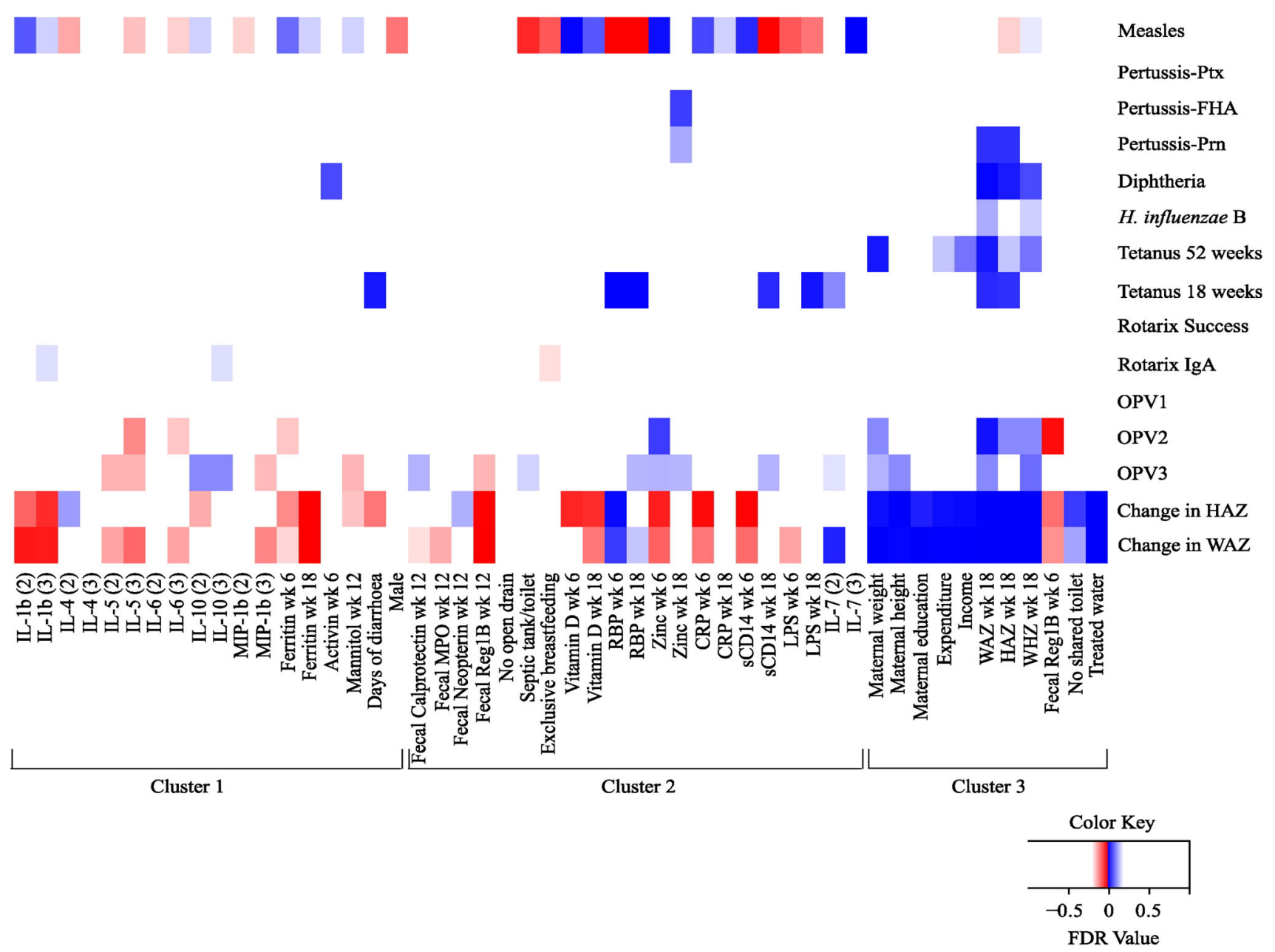

Fig. 3 Relationship of EED and poor performance of oral vaccines against polio and rotavirus. At the time of vaccination, biomarkers of systemic and gut inflammation, micronutrients, and maternal health were measured and correlated with vaccine response. Heatmap of FDR values from univariate linear regression analysis. Biomarkers with a FDR value of 0.2 or below for at least one outcome are depicted on the heatmap. Markers are grouped according to hierarchical cluster results. A positive correlation is indicated by a blue box, and a negative correlation is indicated by a red box. Color patterns reveal associations of biomarkers with outcomes, indicating an improvement or worsening of response. An FDR value close to 0 indicates a strong correlation. Color intensity is indicative of FDR value: darker colors are closer to 0 . Reprinted from Naylor et al., with permission from EBiomedicine ${ }^{85}$ Fecal biomarkers were used to measure EE surrounding the time of vaccination. Myeloperoxidase, calprotectin, and neopterin measured enteric inflammation, and $\alpha-1$ anti-trypsin and reg1B were chosen to represent compromised intestinal epithelial integrity. Systemic inflammation, socioeconomic status, and maternal health were also measured. Nutritional status was assessed by anthropometry and micronutrient levels. Systemic inflammation was tested with inflammatory and regulatory cytokines, acute-phase proteins (CRP, ferritin), and SCD14. The primary outcomes were serum-neutralizing antibody titers to OPV, serum titers to rotavirus IgA, and rotavirus vaccine success. EED was defined noninvasively through biomarkers following a cluster analysis. ${ }^{85}$ HAZ height-for-age $z$ score, WHZ weight-for-height $z$ score, WAZ weight-for-age $z$ score, all defined by comparison to World Health Organization reference growth curves.

enhancing mucosal healing. Such approaches could be used to test whether reducing EE could improve oral vaccine responses. Other interventions designed to manipulate the microbiota, including milk oligosaccharides, ${ }^{89}$ could be evaluated for effects on vaccine responses.

Multiple studies have recently reported inconsistent associations of several EED biomarkers with oral vaccine response. One hypothesis put forward to explain contradictory associations is that an amplified pro-inflammatory response in the mucosa due to EED may increase the immunogenicity of oral vaccines. At this moment, the interpretation of conflicting results from association studies requires an understanding of the relationship with biomarkers of EED, biomarkers of oral vaccine response, and protection from infection. As measuring protection from natural infection is cumbersome to measure, many studies use serum antibody titers to assess response to oral vaccination though it is unclear if this is a suitable proxy for the development of mucosal responses and protection from natural infection. Additionally, recent studies have shown that the timing of peripheral antibody response is critical.

A promising avenue to complement ongoing clinical work with mechanistic studies is the newly developed murine model of EED. The authors found that both malnutrition and specific microbial exposure was required to induce features of human EED including: villous blunting, growth stunting, increased intestinal permeability, and intestinal inflammation. In this model, malnutrition alone altered the intestinal microbiota and increased intestinal permeability, suggesting that undernutrition primes the development of EED by altering the mucosal environment. ${ }^{90}$ This model offers a way to test specific interventions designed to reverse EED or enhance oral vaccine efficacy. 


\section{CONCLUSION}

While there is important evidence from ecological studies that EED and oral vaccine failure are associated, rigorous proof in multiple populations is lacking. If effective therapy were available for any of the domains of pathophysiology of EED, it would be possible to demonstrate that such therapy improves responses to oral vaccines. Such therapy is not yet available, but it is likely that it would also improve child growth and possibly micronutrient status. Immunological understanding of EED is also still at an early stage, with much of it dependent on a very small number of key studies. Current studies will help determine whether the immune alterations in EED are the same as those that impair oral vaccine responses. Evidence from published studies does not give a clear answer. That azithromycin can ameliorate EED, while having no impact on OPV responses, casts doubt on EED being an explanation for poor vaccine responses, but further work is needed, perhaps using prebiotics, probiotics or synbiotics or other tools to heal the mucosal lesion or modulate the microbiota.

\section{ACKNOWLEDGEMENTS}

This work was supported by Bill \& Melinda Gates Foundation (several grants).

\section{ADDITIONAL INFORMATION}

Competing interests: The authors declare no competing interests.

\section{REFERENCES}

1. McDonald, C. M. et al. The effect of multiple anthropometric deficits on child mortality: meta-analysis of individual data in 10 prospective studies from developing countries. Am. J. Clin. Nutr. 97, 896-901 (2013).

2. Kotloff, K. L. et al. Burden and aetiology of diarrhoeal disease in infants and young children in developing countries (the Global Enteric Multicenter Study, GEMS): a prospective, case-control study. Lancet 382, 209-22 (2013).

3. Checkley, W. et al. Asymptomatic and symptomatic cryptosporidiosis: their acute effect on weight gain in Peruvian children. Am. J. Epidemiol. 145, 156-63 (1997).

4. Mølbak, K. et al. Cryptosporidium infection in infancy as a cause of malnutrition: a community study from Guinea-Bissau, west Africa. Am. J. Clin. Nutr. 65, 149-52 (1997).

5. Platts-Mills, J. A. et al. Pathogen-specific burdens of community diarrhoea in developing countries: a multisite birth cohort study (MAL-ED). Lancet Glob. Health 3, e564-75 (2015).

6. Tate, J. E. et al. Global, regional, and national estimates of rotavirus mortality in children under 5 years of age, 2000-2013. Clin. Infect. Dis. 62, S96-S105 (2016).

7. Dangour, A. D. et al. Interventions to improve water quality and supply, sanitation and hygiene practices, and their effects on the nutritional status of children. Cochrane Database Syst. Rev. CD009382 (2013).

8. Gilmartin, A. A. \& Petri, W. A. Exploring the role of environmental enteropathy in malnutrition, infant development and oral vaccine response. Philos. Trans. R. Soc. B 370, 20140143 (2015).

9. Levine, M. M. Immunogenicity and efficacy of oral vaccines in developing countries: lessons from a live cholera vaccine. BMC Biol. 8, 129 (2010).

10. Vesikari, T. et al. Safety and efficacy of a pentavalent human-bovine (WC3) reassortant rotavirus vaccine. N. Engl. J. Med. 354, 23-33 (2006).

11. Mahdi, S. A. et al. Effect of human rotavirus vaccine on severe diarrhea in African infants. N. Engl. J. Med. 362, 289-298 (2010).

12. de Palma, O. et al. Effectiveness of rotavirus vaccination against childhood diarrhoea in El Salvador: case-control study. BMJ 340, c2825 (2010).

13. Zaman, $K$. et al. Efficacy of pentavalent rotavirus vaccine against severe rotavirus gastroenteritis in infants in developing countries in Asia: a randomised, doubleblind, placebo-controlled trial. Lancet 376, 615-623 (2010).

14. Hird, T. R. \& Grassly, N. C. Systematic review of mucosal immunity induced by oral and inactivated poliovirus vaccines against virus shedding following oral poliovirus challenge. PLoS Pathog. 8, e1002599 (2012).

15. Parker, E. P., Kampmann, B., Kang, G. \& Grassly, N. C. Influence of enteric infections on response to oral poliovirus vaccine: a systematic review and metaanalysis. J. Infect. Dis. 210, 853-64 (2014).

16. Ahmed, T., Svennerholm, A.-M., Al Tarique, A., Sultana, G. N. N. \& Qadri, F. Enhanced immunogenicity of an oral inactivated cholera vaccine in infants in Bangladesh obtained by zinc supplementation and by temporary withholding breast-feeding. Vaccine 27, 1433-1439 (2009).
17. Kaufman, D. R. et al. Vitamin A deficiency impairs vaccine-elicited gastrointestinal immunity. J. Immunol. 187, 1877-1883 (2011).

18. Qadri, F., Bhuiyan, T., Sack, D. \& Svennerholm, A. Immune responses and protection in children in developing countries induced by oral vaccines. Vaccine 31, 452-460 (2013).

19. Nordgren, J. et al. Both Lewis and secretor status mediate susceptibility to rotavirus infections in a rotavirus genotype-dependent manner. Clin. Infect. Dis. 59, 1567-73 (2014).

20. Muhsen, K., Pasetti, M. F., Reymann, M. K., Graham, D. Y. \& Levine, M. M. Helicobacter pylori infection affects immune responses following vaccination of typhoid-naive US adults with attenuated Salmonella typhi oral vaccine CVD 908htrA. J. Infect. Dis. 209, 1452-8 (2014).

21. Jiang, N. M. et al. Early life inflammation and neurodevelopmental outcome in Bangladeshi infants growing up in adversity. Am. J. Trop. Med. Hyg. 97, 974-979 (2017).

22. Black, R. E. et al. Maternal and child undernutrition and overweight in low-income and middle-income countries. Lancet 382, 427-51 (2013).

23. Watanabe, K. \& Petri, W. A. Environmental enteropathy: elusive but significant subclinical abnormalities in developing countries. EBioMedicine 10, 25-32 (2016).

24. Manary, M. J. et al. Perturbed zinc homeostasis in rural 3-5-y-old Malawian children is associated with abnormalities in intestinal permeability attributed to tropical enteropathy. Pediatr. Res. 67, 671-675 (2010).

25. Kelly, P. et al. Responses of small intestinal architecture and function over time to environmental factors in a tropical population. Am. J. Trop. Med. Hyg. 70, 412-419 (2004).

26. Lindenbaum, J., Gerson, C. D. \& Kent, T. H. Recovery of small-intestinal structure and function after residence in the tropics. I. Studies in Peace Corps volunteers. Ann. Intern. Med. 74, 218-222 (1971).

27. Gerson, C. D., Kent, T. H., Saha, J. R., Siddiqi, N. \& Lindenbaum, J. Recovery of small-intestinal structure and function after residence in the tropics. II. Studies in Indians and Pakistanis living in New York City. Ann. Intern. Med. 75, 41-48 (1971).

28. Wood, G. M., Gearty, J. C. \& Cooper, B. T. Small bowel morphology in British Indian and Afro-Caribbean subjects: evidence of tropical enteropathy. Gut 32, 256-9 (1991).

29. Grassly, N. et al. The effect of azithromycin on the immunogenicity of oral poliovirus vaccine: a double-blind randomized placebo-controlled trial in seronegative Indian infants. Lancet Infect. Dis. 16, 905-14 (2016).

30. Louis-Auguste, J. et al. High dose multiple micronutrient supplementation improves villous morphology in environmental enteropathy without HIV enteropathy: results from a double-blind randomised placebo controlled trial in Zambian adults. BMC Gastroenterol. 14, 15 (2014).

31. Wang, A. Z. et al. A combined intervention of zinc, multiple micronutrients, and albendazole does not ameliorate environmental enteric dysfunction or stunting in rural Malawian children in a double-blind randomized controlled trial. J. Nutr. 147, 97-103 (2017).

32. Semba, R. D. et al. Child stunting is associated with low circulating essential amino acids. EBioMedicine 6, 246-252 (2016).

33. Semba, R. D. et al. Low serum $\omega-3$ and $\omega-6$ polyunsaturated fatty acids and other metabolites are associated with poor linear growth in young children from rural Malawi. Am. J. Clin. Nutr. 106, 1490-1499 (2017).

34. Blanton, L. V. et al. Gut bacteria that prevent growth impairments transmitted by microbiota from malnourished children. Science 351, aad3311 (2016).

35. Kristensen, K. H. S. et al. Gut microbiota in children hospitalized with oedematous and non-oedematous severe acute malnutrition in Uganda. PLoS Negl. Trop. Dis. 10, e0004369 (2016).

36. Smith-Brown, P., Morrison, M., Krause, L. \& Davies, P. S. Mothers' secretor status affects development of children's microbiota composition and function: a pilot study. PLOS ONE 11, e0161211 (2016).

37. Kelly, P. et al. Endomicroscopic and transcriptomic analysis of impaired barrier function and malabsorption in environmental enteropathy. PLoS Negl. Trop. Dis. 10, e0004600 (2016).

38. Amadi, B. et al. Impaired barrier function and autoantibody generation in malnutrition enteropathy in Zambia. EBioMedicine 22, 191-199 (2017).

39. Menzies, I. et al. Geography of intestinal permeability and absorption. Gut 44, 483-489 (1999).

40. Owino, V. et al. Environmental enteric dysfunction and growth failure/stunting in global child health. Pediatrics 138, e20160641 (2016).

41. Trehan, I., Kelly, P., Shaikh, N. \& Manary, M. J. New insights into environmental enteric dysfunction. Arch. Dis. Child. 101, 741-4 (2016).

42. Denno, D. M., Tarr, P. I. \& Nataro, J. P. Environmental enteric dysfunction: a case definition for intervention trials. Am. J. Trop. Med. Hyg. https://doi.org/10.4269/ ajtmh.17-0183 (2017).

43. Faubion, W. A. et al. A lactulose, rhamnose assay of intestinal permeability in the assessmentof environmental enteric dysfunction. BMJ Glob. Health 1, e000066 (2016). 
44. Lee, G. O. et al. Infant nutritional status, feeding practices, enteropathogen exposure, socioeconomic status, and illness are associated with gut barrier function as assessed by the lactulose mannitol test in the MAL-ED birth cohort. Am. J. Trop. Med. Hyg. 97, 281-290 (2017).

45. Ahmad, R., Sorrell, M. F., Batra, S. K., Dhawan, P. \& Singh, A. B. Gut permeability and mucosal inflammation: bad, good or context dependent. Mucosal Immunol. 10, 307-317 (2017).

46. Gunzel, D. \& Yu, A. S. L. Claudins and the modulation of tight junction permeability. Physiol. Rev. 93, 525-569 (2013).

47. Turner, J. R. Intestinal mucosal barrier function in health and disease. Nat. Rev. Immunol. 9, 799-809 (2009).

48. Menard, S., Cerf-Bensussan, N. \& Heyman, M. Multiple facets of intestinal permeability and epithelial handling of dietary antigens. Mucosal Immunol. 3, 247-259 (2010).

49. Brenchley, J. M. \& Douek, D. C. Microbial translocation across the GI tract. Annu. Rev. Immunol. 30, 149-73 (2012).

50. Kaonga, P. et al. Direct biomarkers of microbial translocation correlate with immune activation in adult Zambians with environmental enteropathy and hepatosplenic schistosomiasis. Am. J. Trop. Med. Hyg. 97, 1603-1610 (2017).

51. Su, L. et al. Targeted epithelial tight junction dysfunction causes immune activation and contributes to development of experimental colitis. Gastroenterology 136, 551-563 (2009).

52. Korpe, P. S. \& Petri, W. A. Environmental enteropathy: critical implications of a poorly understood condition. Trends Mol. Med. 18, 328-336 (2012).

53. Campbell, D. I., Elia, M. \& Lunn, P. G. Growth faltering in rural Gambian infants is associated with impaired small intestinal barrier function, leading to endotoxemia and systemic inflammation. J. Nutr. 133, 1332-1338 (2003).

54. Veitch, A., Kelly, P., Zulu, I., Segal, I. \& Farthing, M. J. G. Tropical enteropathy: a T cell mediated crypt hyperplastic enteropathy. Eur. J. Gastroenterol. Hepatol. 13, 1175-1181 (2001).

55. Yu, J. et al. Environmental enteric dysfunction includes a broad spectrum of inflammatory responses and epithelial repair processes. Cell. Mol. Gastroenterol. Hepatol. 2, 158-17 (2016).

56. Cheroutre, H., Lambolez, F. \& Mucida, D. The light and dark sides of intestinal intraepithelial lymphocytes. Nat. Rev. Immunol. 11, 445-456 (2011)

57. Abadie, V., Discepolo, V. \& Jabri, B. Intraepithelial lymphocytes in celiac disease immunopathology. Semin. Immunopathol. 34, 551-66 (2012).

58. Chang, F., Mahadeva, U. \& Deere, H. Pathological and clinical significance of increased intraepithelial lymphocytes (IELS) in small bowel mucosa. APMIS 113, 385-399 (2005)

59. Steenholt, J. V. et al. The composition of T cell subtypes in duodenal biopsies are altered in coeliac disease patients. PLoS ONE 12, e0170270 (2017).

60. Casey, K. A. et al. Antigen-independent differentiation and maintenance of effector-like resident memory T cells in tissues. J. Immunol. 188, 4866-75 (2012).

61. Korneychuk, N. et al. Interleukin 15 and $\mathrm{CD}^{+}{ }^{+} \mathrm{T}$ cells cooperate to promote small intestinal enteropathy in response to dietary antigen. Gastroenterology 146, 1017-27 (2014)

62. Swamy, M. et al. Intestinal intraepithelial lymphocyte activation promotes innate antiviral resistance. Nat. Commun. 6, 7090 (2015).

63. Zens, K. D., Connors, T. \& Farber, D. L. Tissue compartmentalization of T cell responses during early life. Semin. Immunopathol. 39, 593-604 (2017).

64. Thome, J. J. C. et al. Early-life compartmentalization of human T cell differentiation and regulatory function in mucosal and lymphoid tissues. Nat. Med. 22, 72-77 (2016).

65. Bouskra, D. et al. Lymphoid tissue genesis induced by commensals through NOD1 regulates intestinal homeostasis. Nature 456, 507-510 (2008).

66. Harris, A. M. et al. Antigen-specific memory B-cell responses to Vibrio cholerae 01 infection in Bangladesh. Infect. Immun. 77, 3850-6 (2009).

67. Alam, M. M. et al. Antigen-specific memory B-cell responses in Bangladeshi adults after one- or two-dose oral killed cholera vaccination and comparison with responses in patients with naturally acquired cholera. Clin. Vaccin. Immunol. 18, 844-50 (2011).

68. Akhtar, M. et al. Kinetics of antibody-secreting cell and fecal IgA responses after oral cholera vaccination in different age groups in a cholera endemic country. Vaccine 35, 321-8 (2017).

69. Takenouchi, N. et al. Retinoic acid enhances the gene expression of human polymeric immunoglobulin receptor (plgR) by TNF-a. Clin. Exp. Immunol. 135, 448-454 (2004).

70. Lundgren, A., Jertborn, M. \& Svennerholm, A.-M. Induction of long term mucosal immunological memory in humans by an oral inactivated multivalent enterotoxigenic Escherichia coli vaccine. Vaccine 34, 3132-40 (2016).

71. Hapfelmeier, S. et al. Reversible microbial colonization of germ-free mice reveals the dynamics of $\lg$ A immune responses. Science 328, 1705-9 (2010).

72. Beale, A. J., Douglas, A. P., Parish, W. E. \& Hobbs, J. R. Impaired IgA responses in coeliac disease. Lancet 1, 1198-1200 (1971).

73. Mawhinney, H. \& Love, A. H. The immunoglobulin class responses to oral poliovaccine in coeliac disease. Clin. Exp. Immunol. 21, 399-406 (1975).
74. Hughes, S. M. et al. Dendritic cell anergy results from endotoxemia in severe malnutrition. J. Immunol. 183, 2818-2826 (2009).

75. Bevins, C. L. \& Salzman, N. H. Paneth cells, antimicrobial peptides and maintenance of intestinal homeostasis. Nat. Rev. Microbiol. 9, 356-368 (2011).

76. Auriti, C. et al. Mannose-binding lectin: biologic characteristics and role in the susceptibility to infections and ischemia-reperfusion related injury in critically ill neonates. J. Immunol. Res. 2017, 7045630 (2017).

77. Dhaliwal, W., Bajaj-Elliott, M. \& Kelly, P. Intestinal defensin gene expression in human populations. Mol. Immunol. 40, 469-475 (2003).

78. Dhaliwal, W. et al. Intestinal antimicrobial gene expression: impact of micronutrients in malnourished adults during a randomised trial. J. Infect. Dis. 202, 971-978 (2010).

79. Biragyn, A. et al. Mediators of innate immunity that target immature, but not mature, dendritic cells induce antitumor immunity when genetically fused with nonimmunogenic tumor antigens. J. Immunol. 167, 6644-6653 (2001).

80. Grigat, J., Soruri, A., Forssman, U., Riggert, J. \& Zwirner, J. Chemoattraction of macrophages, $\mathrm{T}$ lymphocytes, and mast cells is evolutionarily conserved within the human beta-defensin family. J. Immunol. 179, 3958-3965 (2007).

81. Garlapati, S. et al. Strategies to link innate and adaptive immunity when designing vaccine adjuvants. Vet. Immunol. Immunopathol. 128, 184-191 (2009).

82. Becker-Dreps, S. et al. The association between fecal biomarkers of environmental enteropathy and rotavirus vaccine response in Nicaraguan infants. Pediatr. Infect. Dis. J. 36, 412-416 (2017).

83. Haque, R. et al. Oral polio vaccine response in breast fed infants with malnutrition and diarrhoea. Vaccine 32, 478-482 (2014).

84. Perez-Schael, I. et al. Efficacy of the human rotavirus vaccine RIX4414 in malnourished children. J. Infect. Dis. 196, 537-40 (2007).

85. Naylor, C. et al. Environmental enteropathy, oral vaccine failure and growth faltering in infants in Bangladesh. EBioMedicine 2, 1759-66 (2015).

86. Taniuchi, M. et al. Impact of enterovirus and other enteric pathogens on oral polio and rotavirus vaccine performance in Bangladeshi infants. Vaccine 34, 3068-75 (2016)

87. Uddin, M. I. et al. Biomarkers of environmental enteropathy are positively associated with immune responses to an oral cholera vaccine in Bangladeshi children. PLoS Negl. Trop. Dis. 10, e0005039 (2016).

88. Panigrahi, P. et al. A randomized synbiotic trial to prevent sepsis among infants in rural India. Nature 548, 407-412 (2017).

89. Charbonneau, M. R. et al. Sialylated milk oligosaccharides promote microbiotadependent growth in models of infant undernutrition. Cell 164, 1-13 (2016).

90. Brown, E. et al. Diet and specific microbial exposure trigger features of environmental enteropathy in a novel murine model. Nat. Commun. 6, 7806 (2015).

91. Kosek, M. et al. Fecal markers of intestinal inflammation and permeability associated with the subsequent acquisition of linear growth deficits in infants. Am. J. Trop. Med. Hyg. 88, 390-6 (2013).

92. Wu, R. L. et al. Gluten-induced symptoms in diarrhea-predominant irritable bowel syndrome are associated with increased myosin light chain kinase activity and claudin-15 expression. Lab. Invest. 97, 14-23 (2017).

93. Lima, A. A. M. et al. Intestinal barrier function and weight gain in malnourished children taking glutamine supplemented enteral formula. J. Pediatr. Gastroenterol. Nutr. 40, 28-35 (2005).

94. Donowitz, J. R. et al. Small intestine bacterial overgrowth and environmental enteropathy in Bangladeshi children. mBio 7, e02102-15 (2016).

95. Jones, K. D. et al. Mesalazine in the initial management of severely acutely malnourished children with environmental enteric dysfunction: a pilot randomized controlled trial. BMC Med. 12, 133 (2014).

96. Mayneris-Perxachs, J. et al. Urinary N-methylnicotinamide and $\beta$-aminoisobutyric acid predict catch-up growth in undernourished Brazilian children. Nat. Sci. Rep. 6, 19780 (2016)

97. Rubio, C. A. The natural antimicrobial enzyme lysozyme is up-regulated in gastrointestinal inflammatory conditions. Pathogens 3, 73-92 (2014).

Open Access This article is licensed under a Creative Commons Attribution 4.0 International License, which permits use, sharing, adaptation, distribution and reproduction in any medium or format, as long as you give appropriate credit to the original author(s) and the source, provide a link to the Creative Commons license, and indicate if changes were made. The images or other third party material in this article are included in the article's Creative Commons license, unless indicated otherwise in a credit line to the material. If material is not included in the article's Creative Commons license and your intended use is not permitted by statutory regulation or exceeds the permitted use, you will need to obtain permission directly from the copyright holder. To view a copy of this license, visit http://creativecommons. org/licenses/by/4.0/

(c) The Author(s) 2018 